\title{
Anti-proliferative effects of progesterone antagonists in the primate endometrium: a potential role for the androgen receptor
}

\author{
Robert M. Brenner ${ }^{1}$, Ov D. Slayden ${ }^{1}$ and Hilary O. D. Critchley ${ }^{2}$ \\ ${ }^{1}$ Oregon National Primate Research Center, Beaverton, OR 97006, USA; and ${ }^{2}$ Centre for \\ Reproductive Biology, University of Edinburgh, Edinburgh EH3 9EW, UK
}

\begin{abstract}
In women and non-human primates, treatment with anti-progestins suppresses oestrogendependent mitotic activity in the endometrial glands. This anti-proliferative effect is paradoxical, because anti-progestins do not bind to the oestrogen receptor. Although this phenomenon has been termed a 'non-competitive anti-oestrogenic effect', it does not occur in all species or in other regions of the primate reproductive tract, so is best referred to as an 'endometrial anti-proliferative effect'. The abundance of androgen receptors is greatly increased by anti-progestin treatment, especially in the glandular epithelium in non-human primates and women. Such an increase could lead to an enhancement of androgen action in the endometrium. As androgens suppress oestrogen-dependent endometrial proliferation, the increased abundance of androgen receptors could mediate the anti-proliferative effects of anti-progestin treatment. This brief review evaluates the implications of these findings.
\end{abstract}

This review focuses specifically on the endometrial antiproliferative effects of progesterone antagonists. More extensive reviews of the chemistry and physiological effects of progesterone antagonists have been published by Spitz and Robbins (1998) and Chwalisz et al. (2000). The first effective anti-progestin was mifepristone (RU486), and the literature on this compound has been reviewed several times (Baulieu et al., 1987; Baulieu, 1991). Several other antiprogestins have been developed, including onapristone, ZK 137316 and ZK 230211 (Schering AG, Berlin), which have greater potency and higher specificity for the progesterone receptor (PR). In addition, mixed agonistantagonists such as J1042 and J867 (Schering AG), known as either mesoprogestins or progesterone receptor modulators (PRMs), have been developed (Chwalisz et al., 2000). PRMs can block progesterone action, but in the absence of progesterone can act as agonists. Both PRMs and antiprogestins have many potentially important clinical uses, and chronic low dose treatment with anti-progestins has been proposed for management of aberrant endometrial bleeding, endometriosis, mammary cancer and contraception (Cadepond et al., 1997; Baird, 2000). However, concern has been expressed that anti-progestins might block the ability of progesterone to oppose oestrogen action in the endometrium, resulting in hyperplasia.

As a counter to this concern, several studies have indicated that anti-progestins have the unexpected ability to block endometrial proliferation, even during oestrogenonly treatment, although the mechanism of this action has

Email: brennerr@ohsu.edu not been fully explained. Wolf et al. (1989) were the first to report that high doses of RU486 had an anti-endometrial effect in ovariectomized, oestrogen-treated macaques. As RU486 does not bind to the oestrogen receptor, these authors called this action a 'non-competitive anti-oestrogenic effect'.

\section{Endometrial anti-proliferative effects of anti- progestins in the primate uterus}

The effects of various regimens of RU486 and some Schering anti-progestins, such as ZK 137316 , on the endometrium of rhesus macaques has been reviewed by Chwalisz et al. (2000). All these anti-progestins induced increases in endometrial oestrogen receptor (ER) and PR, a classical effect of unopposed oestradiol, but they inhibited endometrial mitotic activity, wet mass and thickness. In addition, anti-progestin treatment led to stromal cell atrophy, stromal compaction and hyalinizing degeneration of the spiral arteries, all in the presence of high serum concentrations of oestradiol and increased ER. Similar paradoxical effects were found after long-term treatment of intact cyclic macaques with low doses of RU486 for 8 years (Grow et al., 1998). In studies with ZK 137316 in ovary intact, cyclic animals, the lowest doses allowed normal ovarian cycles, secretion of follicular phase concentrations of oestradiol and normal oviductal differentiation, but an overall suppression of endometrial growth, including a decrease in glandular cell proliferation, assessed on the basis of both mitotic counts and DNA synthesis. These data indicate that the endometrium is much more sensitive to the negative effects of anti-progestins than are other tissues that 
contain PR. Low doses of RU486 administered chronically to women also inhibited glandular mitosis and induced stromal compaction in the endometrium (Cameron et al., 1996).

Anti-proliferative and apoptotic effects of anti-progestins have also been observed in vitro in various breast cancer cell lines, and these effects have been referred to as receptor-mediated 'cytostatic and cytotoxic' effects. Conceivably, RU486 might prove a valuable drug for treatment of breast cancer because of such direct antiproliferative effects (Horwitz, 1992). Mifepristone has antioxidant properties and it has been proposed that the anti-proliferative action of RU486 may involve antioxidants blocking oxidative changes in low density lipoproteins that are important to the growth of human endometrium (Murphy et al., 2000). However, the failure of known anti-oxidants such as vitamin $\mathrm{E}$ to have similar antiproliferative effects (Murphy et al., 2000) raises doubts about the generality of this hypothesis. Moreover, in macaques, anti-progestins do not appear to cause any antiproliferative or anti-differentiative effects in the oviductal or vaginal epithelium, and do not reduce oestrogen-dependent bone mineral density in either women or macaques. In addition, in rodents treated with anti-progestin, oestradiol action in the endometrium is enhanced rather than suppressed, as indicated by increases in cell height and mitotic activity of the uterine luminal epithelium (Chwalisz et al., 1998). In summary, the non-competitive antioestrogenic effects of chronic low dose anti-progestin therapy appear to be tissue- and species-specific, are most evident in the primate endometrium, and are best referred to as endometrial anti-proliferative effects. A full understanding of the mechanisms underlying these paradoxical effects has not yet been reached.

\section{Is the androgen receptor involved in the endometrial anti-proliferative effect?}

There is substantial evidence that exogenous androgens can inhibit the endometrium. Okon et al. (1998) suggested that in women with recurrent miscarriages, high androgen concentrations may specifically antagonize oestrogen action directly in the endometrium. Testosterone and danazol directly inhibit human endometrial cell proliferation in tissue culture in which the medium contains phenol red in amounts adequate to provide an oestrogenic stimulus (Rose et al., 1988). Other studies show that androstenedione inhibits human endometrial cell growth and secretory activity in vitro and that androgen receptors are present in these cultured endometrial epithelial cells. Moreover, when cyproterone acetate (an androgen receptor antagonist) is added to the cultures, the effects of the androgens are blocked (Tuckerman et al., 2000). Therapeutic progestogens that are highly androgenic result in endometrial atrophy. For example, marked endometrial atrophy is a feature of the endometrial response among women using a levonorgestrelreleasing intrauterine system (Critchley et al., 1998). As the suppressive effects of androgens on endometrial growth resemble the atrophic effects induced by anti-progestins, the possibility that the androgen receptor (AR) plays a role in anti-progestin action in human and non-human primate endometrium was evaluated using in situ hybridization, immunocytochemistry and ligand binding assays to assess changes in the endometrial AR during anti-progestin treatment (Slayden et al., 2001).

Human endometrial samples from three groups of patients were available from three previously published studies. The first group consisted of fertile women who had been sampled during the proliferative, early mid- and late secretory phases of the normal menstrual cycle for studies of oestrogen receptor isoform expression (Critchley et al., 2001).

The second group consisted of women with regular menstrual cycles treated either with placebo capsules or capsules of $2 \mathrm{mg}$ mifepristone each day for 30 days. Such chronic, low dose treatment suppresses endometrial maturation and makes successful implantation unlikely (Batista et al., 1992; Cameron et al., 1996). The third group consisted of women who were treated with $200 \mathrm{mg}$ oral mifepristone (or vehicle) 2 days after the ovulatory surge of LH and sampled 4-6 days later. Acute administration of a high dose of mifepristone $(200 \mathrm{mg})$ in the early luteal phase also retards development of a secretory endometrium (Cameron et al., 1997). All human endometrial samples were obtained with a Pipelle endometrial sampler.

Ovariectomized rhesus macaques were treated with implants of oestradiol for 2 weeks and then for 2 further weeks with either oestradiol alone, oestradiol plus RU486, oestradiol plus progesterone, or oestradiol plus progesterone plus RU486. Untreated, ovariectomized animals served as controls. Reproductive tract tissues were collected by midventral laparotomy at the end of treatment. Some animals were also treated with oestradiol and various doses of ZK 137316 (Schering AG).

All work with macaques received ethical approval from the Institutional Animal Care and Use Committee of the Oregon National Primate Research Center.

\section{Effects of anti-progestins in rhesus macaques}

In macaques, oestradiol significantly increased AR binding capacity (assessed with a radioactive androgen) above untreated controls, and oestradiol plus RU486 treatment increased binding significantly further (Fig. 1). Oestradiol plus progesterone decreased AR binding, and oestradiol plus progesterone plus RU486 treatment caused an intermediate increase in AR binding. In macaques treated with oestradiol alone, all immunocytochemical staining for AR was localized to the stroma, and the glands were negative (Fig. 2). In macaques treated with oestradiol plus RU486, the stromal AR signal was enhanced and strong AR staining became evident in the glandular epithelium (Fig. 2). In situ hybridization for mRNA encoding AR showed similar patterns: after treatment with oestradiol alone, hybridization 


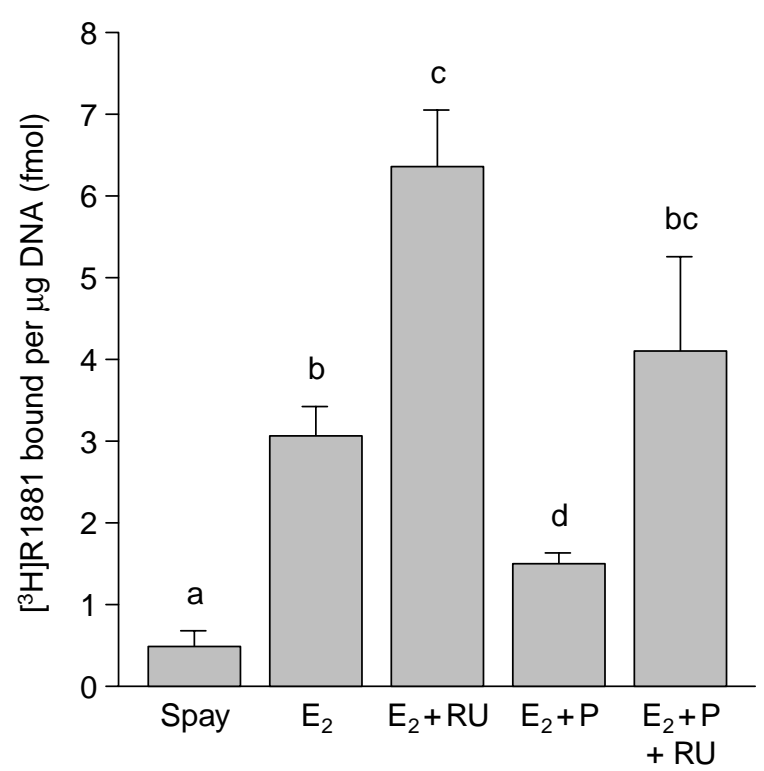

Fig. 1. Endometrial total androgen receptor (AR) concentrations (mean $\pm \mathrm{SE}$ ) measured by $\left[{ }^{3} \mathrm{H}\right] \mathrm{R} 1881$ binding assay in untreated (spay) macaques, and macaques treated with oestradiol $\left(E_{2}\right)$, oestradiol plus RU486 (RU), oestradiol plus progesterone $(\mathrm{P})$, and

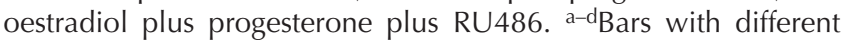
letters are significantly different $(P<0.05)$. (Adapted from Slayden et al., 2001.)

signals were evident only in the stroma, but after treatment with oestradiol plus RU486, the hybridization signal increased in the stroma and became very strong over the glandular epithelium (Slayden et al., 2001). No evidence for AR expression was obtained by either immunocytochemistry or in situ hybridization in the vascular endothelium or smooth muscle of the spiral arteries under normal cyclic conditions or after anti-progestin treatment. The effects with ZK 137316 were similar to those obtained using RU486.

\section{Effects of anti-progestins in women}

In women, during the natural menstrual cycle, immunocytochemical staining for AR also predominated in the stroma. The signal was greatest in the proliferative, early and mid-secretory phases and weakest in the late secretory phase. As in macaques, mifepristone treatment resulted in increased staining in the stroma and a marked increase in staining for AR in the glandular epithelium (Fig. 2). The same anti-AR antibody (F39.4; Biogenex, San Ramon, CA) was used to evaluate both human and macaque tissues. The increase in AR staining induced by mifepristone was evident after either treatment with $2 \mathrm{mg}$ per day for 30 days or after a single oral dose of $200 \mathrm{mg}$. In summary, endometrial AR was highest in the stroma during the human proliferative phase (or during oestradiol treatment in macaques), and lowest during the late secretory phase in women (or after oestradiol plus progesterone treatment in macaques). In both species, RU486 markedly increased AR expression in the glands and enhanced its expression in the stroma.

These data show that during the normal menstrual cycle, in both women and macaques, endometrial AR is primarily stromal in distribution. Similar findings have been reported previously in the human (Mertens et al., 1996) and rhesus macaque endometrium (Adesanya et al., 1999). Apparao et al. (2002) reported that, in endometrial biopsies from women with polycystic ovary syndrome (PCOS), the glandular and luminal epithelia had much stronger immunocytochemical staining for AR than they did in similar specimens from women without PCOS. These authors suggested that the combination of increased androgens and glandular AR in the endometrium of women with PCOS could play a major role in the infertility associated with this syndrome.

\section{Working hypothesis for the endometrial anti- proliferative effect}

In summary, during the natural menstrual cycle in normal women, any effects of androgens on the glands or vascular elements in the endometrium would be mediated indirectly through stromal AR. However, after treatment with antiprogestins (and perhaps in women with PCOS), androgens may have direct effects on the glands through the greatly increased AR in glandular cells. Any effects of oestrogen that are mediated by ER in the glands might then be counteracted by direct effects of androgens mediated by AR in the same cells. In addition, a large body of evidence indicates that oestrogen-dependent growth factors that are made by the stroma are essential for mitosis in the glands (Cooke et al., 1997). Anti-progestins, by increasing AR in stromal cells, might lead to suppression of such factors by androgens. Whether these are the effects of testosterone, dihydrotestosterone, androstenedione or some other androgen, and whether the androgen source is the endometrial tissue or the systemic circulation is not known. It is possible that AR is activated through cross-talk by cellular factors that enhance its phosphorylation state (Ikonen et al., 1994), but it is not known whether this occurs in the primate endometrium. All of these considerations demand further research.

The precise mechanism through which anti-progestin treatment increases AR during treatment with oestradiol is not clear, but our previous studies and those of others showed that anti-progestin treatment also increases ER and PR. Our current view is similar to that expressed by Chwalisz et al. (1991), who found an increase in ER after treatment with the anti-progestin, onapristone, in the endometrium of ovariectomized rabbits. These authors stated that: 'The increase in ER and ER mRNA concentrations after onapristone treatment in ovariectomized, oestradioltreated animals suggests that this anti-gestagen abolished an inhibitory action of the unoccupied PR on ER biosynthesis'. 


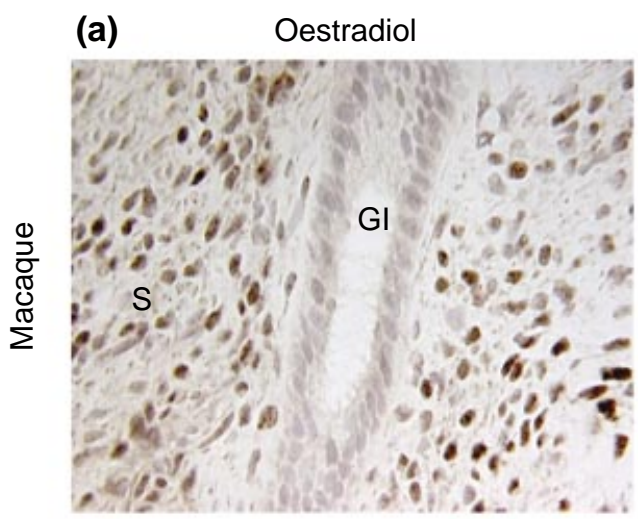

(b) Oestradiol + RU486

(c)

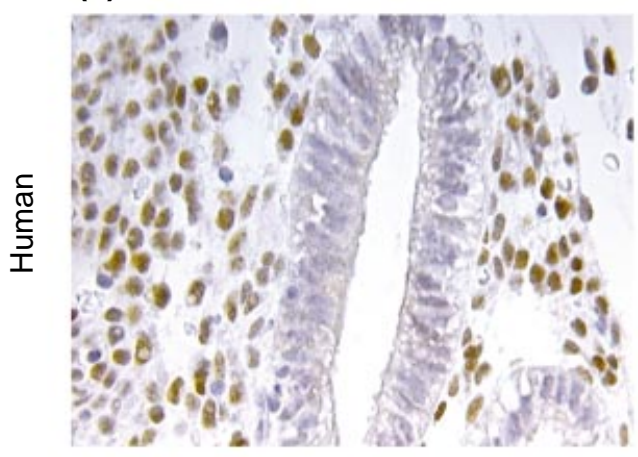

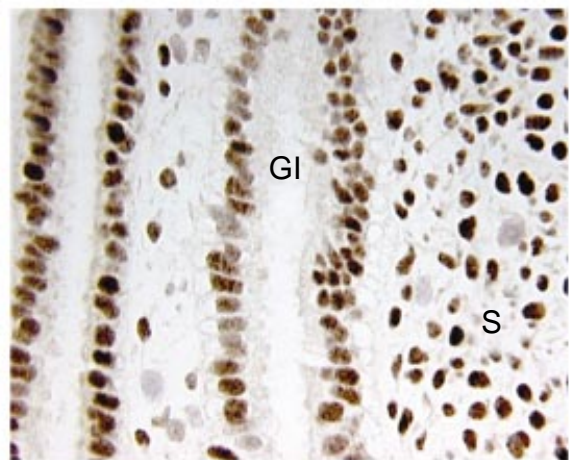

(d) $\quad 200 \mathrm{mg} \mathrm{RU} 486$

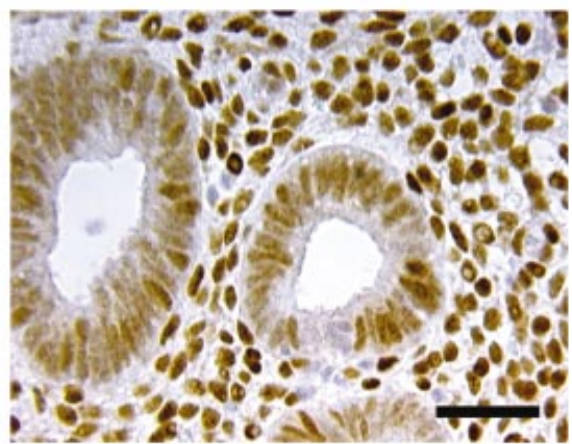

Fig. 2. Androgen receptor $(A R)$ immunostaining in the endometrium of $(a, b)$ macaques and $(c, d)$ women. (a) In macaques treated with oestradiol alone, only the stromal compartment (S) shows definite AR staining; the glands $(\mathrm{Gl})$ are negative for AR. (b) Treatment with oestradiol plus $R U 486$ resulted in a striking upregulation of AR in the glands and further increased stromal AR staining compared with that observed after treatment with oestradiol alone. (c) Human endometrium control sample, stained for AR. Only the stroma is positively stained. (d) Human endometrium after treatment with $200 \mathrm{mg}$ mifepristone (RU486). Both glands and stroma are positively stained for AR. Scale bar represents $50 \mu \mathrm{m}$. (Adapted from Slayden et al., 2001.)

This view implies that unoccupied PR (in the absence of progesterone) has an inhibitory or 'braking' effect on ER synthesis. When this brake is removed by an anti-progestin, in the presence of oestradiol, the amounts of ER, PR and AR increase, as oestradiol can increase the expression of all these molecules. Androgens may also play a role in increasing AR after anti-progestin treatment as androgens enhance the biosynthesis of endometrial AR (Adesanya et al., 1999; Apparao et al., 2002).

How these increases in ER, PR and AR at the molecular level lead to mitotic inhibition and endometrial atrophy at the cellular and organ levels remains unclear. One of the keys to understanding these effects lies in the increased glandular AR, because AR is normally not expressed (or only minimally expressed) in the glandular epithelium. Stated simply, our hypothesis is that increases in endometrial $A R$, induced by anti-progestin treatment, allow androgens to suppress oestrogen-dependent glandular mitosis.

\section{Conclusion}

Paradoxically, anti-progestins block oestradiol action on glandular proliferation and suppress the overall growth of the primate endometrium. The mechanism involved in this endometrial anti-proliferative effect is not known, but the data reviewed above indicate that androgens play a previously unsuspected role.

As the androgen receptor is normally expressed primarily in the stroma, an increase in intracellular androgens bound to the glandular epithelium would represent a highly abnormal state that could suppress the metabolic rate and interfere with the mitotic machinery of the glandular cells. In addition, epithelial AR can directly mediate antiproliferative effects of androgens, at least in vitro (Tuckerman et al., 2000), and, because stromal AR is also increased, an enhanced sensitivity of the stroma to androgens could block any oestrogen-dependent, stromalepithelial interactions involved in glandular mitosis. The 
(a) Effects of oestradiol alone

Lumen

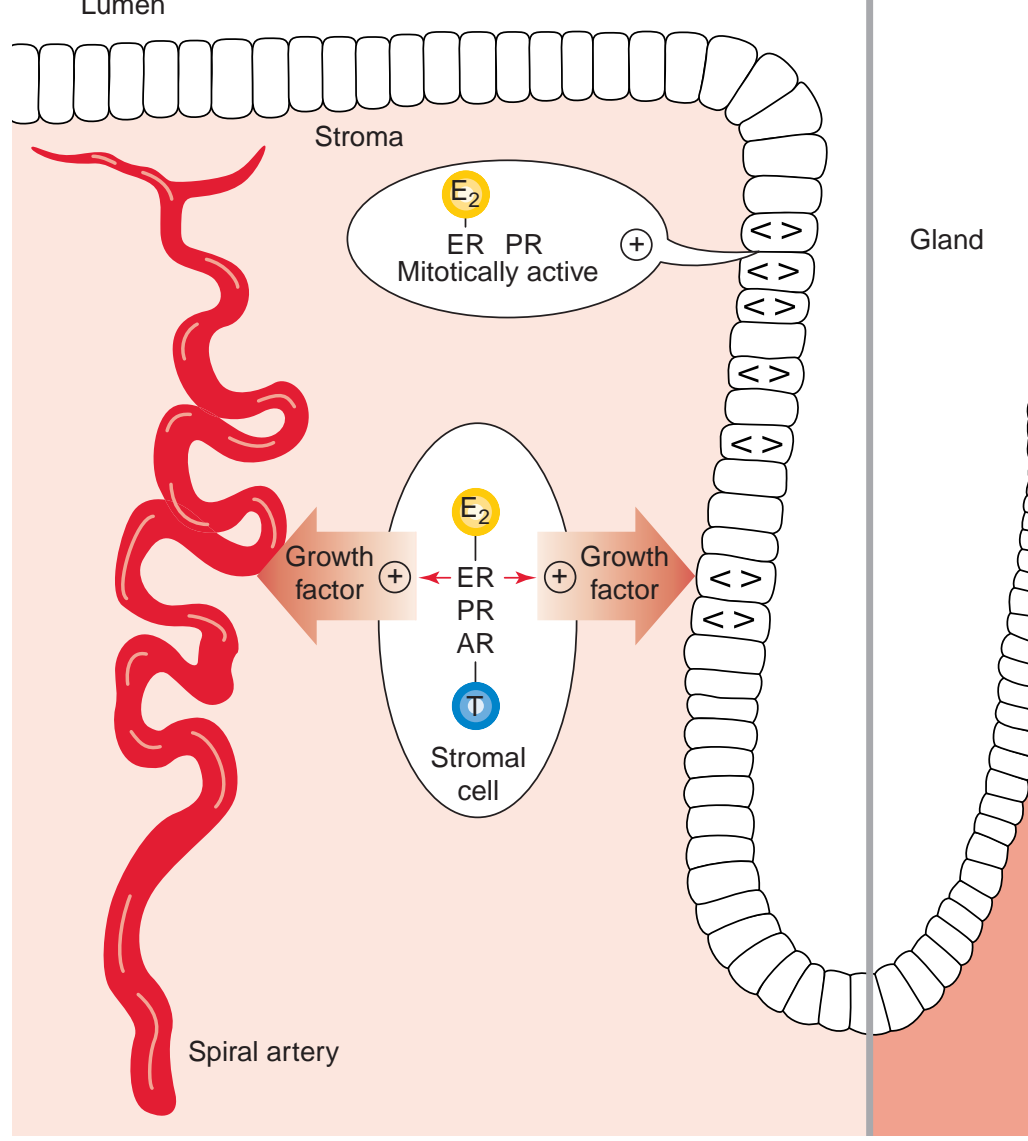

(b) Effects of oestradiol + anti-progestin

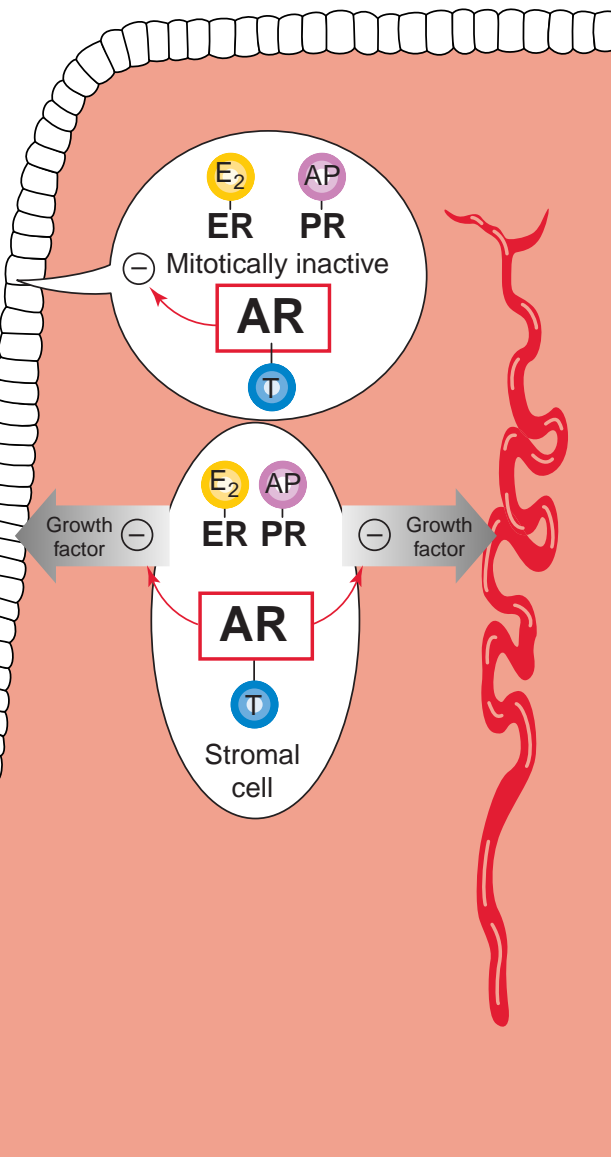

Fig. 3. Hypothetical and known effects of oestradiol administered either (a) alone or (b) combined with an anti-progestin (AP) on the primate endometrium. Growth factor means any stimulatory factor, including cytokines and chemokines. Anti-progestin treatment induces stromal compaction in the endometrium and this is symbolized by the darker background colour in the right panel. (a) In stromal cells, oestradiol $\left(E_{2}\right)$ acts through oestrogen receptor (ER) to induce growth factors that have two effects: stimulation of epithelial mitosis and growth of the spiral arteries (which grow in the follicular phase under oestradiol dominance, and grow further in the luteal phase under progesterone dominance). The androgen receptor (AR) is present at low concentrations and can bind androgens but its effects are unknown. In epithelial cells, oestradiol acts through ER to enhance metabolic activity, as evidenced by the tall, hypertrophied, columnar cells, and presumably to facilitate mitosis. AR is absent or present in very low concentrations in epithelial cells. The diagram refers only to oestradiol-dependent arterial growth; the paradoxical effect of antiprogestins is that they block oestradiol-dependent as well as progesterone-dependent growth. (b) Addition of anti-progestins to oestradiol treatment leads to a great increase in AR, as well as ER and progesterone receptor (PR), in both epithelial and stromal cells. In stromal cells, the increases in androgen ( $\mathrm{T}$ ) action may overcome oestradiol action, inhibit growth factor production and result in two effects: suppression of epithelial mitosis and blockade of oestradiol-dependent spiral artery growth. In epithelial cells, the increases in androgen action may overcome oestradiol action and reduce metabolic activity, as evidenced by the short, atrophied cuboidal cells; this inhibition would favour mitotic suppression.

lack of AR expression in the vascular endothelium and smooth muscle walls also indicates that the atrophy and degenerative changes induced in the spiral arteries by antiprogestin treatment are indirectly mediated. Androgens may play a role in this atrophy and degeneration by suppressing biosynthesis of any oestrogen-dependent, stromal growth factors necessary for arterial growth (Fig. 3).

A cautionary note on this hypothesis is necessary. In addition to the blockade of glandular cell mitosis, anti- progestins also induce severe compaction of the endometrial stroma, an increase in epithelial apoptosis and severe degenerative changes in the spiral arteries. Arterial atrophy and degeneration may greatly reduce endometrial blood flow, and this alone, through simple nutritional deprivation, could contribute to the endometrial atrophy that occurs in macaques during anti-progestin treatment. The matter is certainly complex and it is not known whether increased androgen action through increased AR can 
induce all the endometrial changes induced by antiprogestins. Fortunately, a variety of androgens and antiandrogens is available as tools to conduct experimental studies, so the role of androgens in the paradoxical effects of anti-progestins in the endometrium should eventually be more thoroughly understood. We hope that the novel hypothesis put forward in this brief review will stimulate research along these lines. The anti-progestins and PRMs have tremendous potential for treatment of various diseases, and their use in clinical medicine is increasing. A more complete understanding of their mode of action should greatly benefit women's health.

The authors wish to thank Kunie Mah, Xiao Jing Nie (Oregon) and Teresa Henderson (Edinburgh) for technical assistance and Angela Adler (Oregon) for word processing. This project was supported by grants DAMD15-96-C-6096 and NIH RR-00163 (US), Wellbeing (UK) and a Burroughs Wellcome Fellowship to R. M. Brenner. Figures 1 and 2 were reproduced with permission from the following: Slayden et al. (2001) Progesterone antagonists increase androgen receptor expression in the rhesus macaque and human endometrium Journal of Clinical Endocrinology and Metabolism 86 2668-2679. Copyright owner, The Endocrine Society.

\section{References}

Key references are identified by asterisks.

Adesanya OO, Zhou J, Wu G and Bondy C (1999) Location and sex steroid regulation of androgen receptor gene expression in rhesus monkey uterus Obstetrics and Gynecology 93 265-270

*Apparao KB, Lovely LP, Gui Y, Lininger RA and Lessey BA (2002) Elevated endometrial androgen receptor expression in women with polycystic ovarian syndrome Biology of Reproduction 66 297-304

Baird DT (2000) Overview of advances in contraception British Medical Bulletin 56 704-716

Batista MC, Cartledge TP, Zellmer AW, Merino MJ, Axiotis CA, Loriaux DL and Nieman LK (1992) Delayed endometrial maturation induced by daily administration of the antiprogestin RU486: a potential new contraceptive strategy American Journal of Obstetrics and Gynecology 167 60-65

Baulieu EE (1991) The antisteroid RU486 Trends in Endocrinology and Metabolism 2 233-239

Baulieu EE, Ulmann A and Philibert D (1987) Contragestion by antiprogestin RU486: a review Archives of Gynecology and Obstetrics 241 73-85

Cadepond F, Ulmann A and Baulieu EE (1997) RU486 (mifepristone): mechanisms of action and clinical uses Annual Reviews of Medicine $\mathbf{4 8}$ 129-156

Cameron ST, Critchley HOD, Thong KJ, Buckley $\mathrm{CH}$, Williams AR and Baird DT (1996) Effects of daily low dose mifepristone on endometrial maturation and proliferation Human Reproduction $112518-2526$

Cameron ST, Critchley HOD, Buckley CH, Kelly RW and Baird DT (1997) Effect of two antiprogestins (mifepristone and onapristone) on endometrial factors of potential importance for implantation Fertility and Sterility 67 1046-1053

Chwalisz K, Hegele-Hartung C, Fritzemeier K-H, Beier HM and Elger W (1991) Inhibition of the estradiol-mediated endometrial gland formation by the antigestagen onapristone in rabbits: relationship to uterine estrogen receptors Endocrinology 129 312-322

Chwalisz K, Stöckemann K, Fritzemeier K-H and Fuhrmann U (1998) Modulation of oestrogenic effects by progesterone antagonists in the rat uterus Human Reproduction Update 4 570-583

*Chwalisz K, Brenner RM, Fuhrmann U, Hess-Stumpp H and Elger W (2000) Antiproliferative effects of progesterone antagonists and progesterone receptor modulators (PRMs) on the endometrium Steroids 65 741-751

Cooke PS, Buchanan DL, Young P, Setiawan T, Brody J, Korach KS, Taylor J, Lubahn DB and Cunha GR (1997) Stromal estrogen receptors mediate mitogenic effects of estradiol on uterine epithelium Proceedings National Academy of Sciences USA 94 6535-6540

Critchley H, Wang H, Jones R, Kelly R, Trudy T, Gebbie A, Buckley C, McNeilly A and Glasier A (1998) Morphological and functional changes in human endometrium following intrauterine levonorgestrel delivery Human Reproduction 13 1218-1224

Critchley HOD, Brenner RM, Henderson TA, Williams K, Nayak NR, Slayden OD, Millar MR and Saunders PTK (2001) Estrogen receptor beta, but not estrogen receptor alpha, is present in the vascular endothelium of the human and nonhuman primate endometrium Journal of Clinical Endocrinology and Metabolism 86 1370-1378

Grow DR, Reece MT, Hsiu JG, Adams L, Newcomb PM, Williams RF and Hodgen GD (1998) Chronic antiprogestin therapy produces a stable atrophic endometrium with decreased fibroblast growth factor: a 1-year primate study on contraception and amenorrhea Fertility and Sterility 69 936-943

Horwitz KB (1992) The molecular biology of RU486: is there a role for antiprogestins in the treatment of breast cancer? Endocrine Reviews 13 146-163

Ikonen T, Palvimo JJ, Kallio PJ, Reinikainen P and Janne OA (1994) Stimulation of androgen-regulated transactivation by modulators of protein phosphorylation Endocrinology 135 1359-1366

Mertens HJ, Heineman MJ, Koudstaal J, Theunissen P and Evers JL (1996) Androgen receptor content in human endometrium European Journal of Obstetrics, Gynecology and Reproductive Biology 70 11-13

Murphy AA, Zhou MH, Malkapuram S, Santanam N, Parthasarathy S and Sidell N (2000) RU486-induced growth inhibition of human endometrial cells Fertility and Sterility 74 1014-1019

Okon MA, Laird SM, Tuckerman EM and Li T-C (1998) Serum androgen levels in women who have recurrent miscarriages and their correlation with markers of endometrial function Fertility and Sterility $69682-690$

Rose GL, Dowsett M, Mudge JE, White JO and Jeffcoate SL (1988) The inhibitory effects of danazol, danazol metabolites, gestrinone, and testosterone on the growth of human endometrial cells in vitro. Fertility and Sterility 49 224-228

*Slayden OD, Nayak NR, Burton KA, Chwalisz K, Cameron ST, Critchley HO, Baird DT and Brenner RM (2001) Progesterone antagonists increase androgen receptor expression in the rhesus macaque and human endometrium Journal of Clinical Endocrinology and Metabolism $\mathbf{8 6}$ 2668-2679

Spitz IM and Robbins A (1998) Mechanism of action and clinical effects of antiprogestins on the non-pregnant uterus Human Reproduction Update 4 584-593

*Tuckerman EM, Okon MA, Li T-C and Laird SM (2000) Do androgens have a direct effect on endometrial function? An in vitro study Fertility and Sterility 74 771-779

*Wolf JP, Hsiu JG, Anderson TL, Ulmann A, Baulieu EE and Hodgen GD (1989) Noncompetitive antiestrogenic effect of RU486 in blocking the estrogen-stimulated luteinizing hormone surge and the proliferative action of estradiol on endometrium in castrate monkeys Fertility and Sterility 52 1055-1060 